Éditorial

\title{
POUR DES CONTRIBUTIONS VARIÉES DANS LA REVUE SIM
}

\author{
François de Corbière, Cécile Godé, Jessie Pallud
}

ESKA | «Systèmes d'information \& management »

2021/2 Volume 26 | pages 3 à 6

ISSN 1260-4984

ISBN 9782747232111

Article disponible en ligne à l'adresse :

https://www.cairn.info/revue-systemes-d-information-etmanagement-2021-2-page-3.htm

Distribution électronique Cairn.info pour ESKA.

(C) ESKA. Tous droits réservés pour tous pays.

La reproduction ou représentation de cet article, notamment par photocopie, n'est autorisée que dans les limites des conditions générales d'utilisation du site ou, le cas échéant, des conditions générales de la licence souscrite par votre établissement. Toute autre reproduction ou représentation, en tout ou partie, sous quelque forme et de quelque manière que ce soit, est interdite sauf accord préalable et écrit de l'éditeur, en dehors des cas prévus par la législation en vigueur en France. Il est précisé que son stockage dans une base de données est également interdit. 


\title{
Éditorial
}

\section{Pour des contributions variées dans la revue SIM}

\author{
François DE CORBIÈRE*, Cécile GODÉ** EJessie PALLUD*** \\ *IMT Atlantique, LEMNA, Nantes, France \\ **Aix Marseille Univ, CRET-LOG, Aix-en-Provence, France \\ ***EM Strasbourg Business School, Université de Strasbourg, HuManiS, France
}

Dans notre précédent éditorial (Pallud et al., 2021), premier de notre série sur les rubriques de la revue Systèmes d'Information et Management, nous avons présenté les caractéristiques attendues des deux formes d'articles les plus plébiscitées par notre communauté, à savoir les articles qui testent ou illustrent empiriquement un cadre théorique dans les rubriques « articles de recherche » et «cas, expériences et pédagogie». Dans ce second éditorial de la série, nous aimerions insister sur le potentiel et l'intérêt de trois autres formes d'articles qui se font plus rares : les autres articles de la rubrique « articles de recherche », les articles de la rubrique « recherche méthodologique » et ceux de la rubrique "opinion ». Les soumissions de ces trois formes d'articles sont en effet moins fréquentes. Elles restent pour autant les bienvenues et nous souhaitons donc expliciter ici nos attendus, afin notamment de permettre aux futurs auteurs de s'assurer que leur projet de publication correspond bien aux caractéristiques attendues.

Dans la rubrique « article de recherche », la très grande majorité des articles publiés sont issus de recherches empiriques et leurs caractéristiques ont été présentées dans le premier éditorial de l'année 2021 (Pallud et al., 2021). Ce ne sont toutefois pas les seuls articles que nous publions dans cette rubrique. En effet, des articles de développement d'une théorie et des revues de littérature sont aussi attendus dans cette rubrique.

Concernant les articles qui visent à développer une théorie sans application ou test empirique, les auteurs se doivent d'être particulièrement vigilants pour mettre en cohérence l'ensemble des éléments qui permettent de garantir l'approche scientifique du développement de la théorie. A ce titre, nous nous permettons une petite infidélité à SIM pour vous recommander vivement la lecture du dernier article de Suzanne Rivard (2020) qui propose une démarche pragmatique avec un modèle en spirale pour construire une théorie. Si les six itérations du modèle (i.e. Erudition, Motivation, Definition, Imagination, Explanation $\&$ Presentation, Contribution) ont toute leur importance, nous insistons particulièrement ici sur celle liée à la définition. Rivard montre que la construction d'une théorie implique des définitions précises 
du phénomène étudié, du type de théorie développée (Gregor, 2006 ; Burton-Jones et al., 2015), du périmètre de la théorie, et de ses construits. En effet, sans illustrations ou tests empiriques, il appartient aux auteurs d'accompagner les évaluateurs et les lecteurs dans la compréhension de leur développement théorique, et cette phase de définition devient un élément fondamental pour la clarté de leur démonstration.

Concernant les revues de littérature, il est nécessaire de définir et expliciter le type de revue de littérature utilisé et la méthodologie mise en ouvre. Ceci est une condition nécessaire pour publier une revue de littérature, mais pas une condition suffisante (Rowe, 2014 ; Templier et Paré, 2015). En effet, un article proposant une revue de littérature doit répondre à une question de recherche qui apporte une contribution, permettant de synthétiser et structurer un champ de recherche pour identifier ses limites et voies de recherche.

La rubrique « recherche méthodologique », créée récemment, accueille des articles qui développent des contributions méthodologiques significatives pour la recherche en management des systèmes d'information. Les articles soumis doivent ainsi discuter des conditions d'application, des limites et des opportunités de méthodologies déjà éprouvées, ou proposer des méthodologies innovantes. Parmi les articles méthodologiques publiés dans SIM avant la création de la rubrique, nous pouvons conseiller bien entendu la lecture du numéro spécial dédié (le numéro 3 de 2018) qui présente cinq articles proposant des méthodologies variées pour la recherche en management des systèmes d'information. À titre d'exemples, on y trouve des analyses sur l'intérêt et la pertinence des méthodes mixtes (Pascal et al., 2018), du fuzzy set QCA (Merminod et Rowe, 2018) ainsi que de la combinaison étude de cas et simulation multi-agents (Habib et de Corbière, 2018).
Dans un numéro courant, Walsh et Renaud (2017) démontrent l'intérêt de combiner deux techniques bibliométriques, l'analyse de co-citation de références et l'analyse de couplage bibliographique de documents, pour interpréter la littérature d'un champ de recherche déjà établi.

Concernant la rubrique " opinion », la revue SIM attend «un article développant une opinion, un avis, un paradoxe et une prospective sur des enjeux susceptibles d'intéresser notre communauté " (Pigneur, 2012, p. 3). Nous recommandons aux auteurs souhaitant soumettre un article dans cette rubrique de s'appuyer sur la recherche de Vaujany et Bussy-Socrate (2018). Une fois la mise en contexte, la problématisation et la revue de la littérature développées, les auteurs se fondent sur des ethnographies virtuelles pour décrire trois modes de diffusion de la rumeur.

Nous espérons que cet éditorial incitera les auteurs à soumettre ce type d'articles, peu plébiscités par notre communauté jusqu'alors, et que les éditoriaux futurs pourront en présenter parmi les articles de numéros. Dans ce numéro 2 de l'année 2021, nous avons trois articles de la rubrique «article de recherche», plus « classiques » en s'appuyant sur une démarche empirique pour développer une contribution au management des systèmes d'information (Pallud et al., 2021).

L'article de Anuragini Shirish, Shirish Srivastava et Imed Boughzala propose d'enrichir littérature sur l'innovation digitale en étudiant un mécanisme créatif mobilisant les technologies digitales existantes pour l'innovation. Avec un test empirique auprès de 178 étudiants, les résultats montrent une influence significative à la fois des caractéristiques de l'agent d'innovation et de l'affordance actualisée de l'innovation pour faciliter l'innovation digitale. En particulier, les auteurs démontrent le rôle prépondérant de l' "ICT enabled design-thinking» sur 
l'innovation digitale et discutent de l'intérêt pour les organisations d'intégrer l'utilisation des technologies digitales pour permettre des approches de créativité structurées et encourager les productions innovantes.

Le deuxième article de ce numéro, coécrit par Hajer Kefi, Ekaterina Besson, Karina Sokolova et Chiraz Aouina-Mejri, s'intéresse à la continuation d'usage des assistants personnels virtuels à travers les générations. Construit sur la théorie des usages et des gratifications et la littérature sur les normes sociales et la vie privée, le modèle est testé auprès de 295 utilisateurs issus des générations Y, X et Baby-boomers. Les résultats démontrent l'influence positive des usages utilitaires et hédoniques, des normes subjectives et de la masse critique sur la continuation d'usage des assistants personnels virtuels. De plus, l'intensité perçue des craintes en matière de vie privée influence négativement la continuation d'usage des assistants personnels virtuels et modère l'influence des normes subjectives. Enfin, l'échantillon de l'étude permet de discuter des différences générationnelles sur ces résultats.

Enfin, Jean-François Berthevas présente une étude empirique pour mieux comprendre les facteurs cognitifs et de socialisation qui influencent les comportements en matière de sécurité de l'information. Il combine deux cadres théoriques, à savoir la théorie de la motivation de protection (PMT) et la théorie du lien social (SBT), pour expliquer le comportement des acteurs en matière de sécurité de l'information. L'étude menée auprès de 430 étudiants montre que les individus ont tendance à développer des comportements en matière de sécurité à la fois pour des raisons personnelles (ex : recherche d'efficacité en matière de gestion des risques, compétences détenues en matière de sécurité, vulnérabilité perçue), mais aussi pour des raisons sociales (notamment lorsque les individus sont impliqués dans la prévention des cyber risques au sein de leur entourage). Les résultats pointent également des différences entre les comportements masculins et féminins, ce qui invite à concevoir des programmes différenciés de sensibilisation aux technologies de la sécurité de l'information.

\section{BIBLIOGRAPHIE}

Burton-Jones, A., McLean, E.R., Monod, E. (2015). "Theoretical perspectives in IS research: From variance and process to conceptual latitude and conceptual fit." European Journal of Information Systems, 24(6), 664-679.

de Vaujany, F. X., Bussy-Socrate, H. (2018). «Des modes de diffusion de la rumeur : une approche par la matérialité ». Systèmes d'Information et Management, 23(4), 9-30.

Gregor, S. (2006). "The nature of theory in information systems." MIS Quarterly, 30(3), 611-642.

Habib, J., et de Corbière, F. (2018). « Proposition d'un design de recherche pour l'analyse des processus complexes et émergents en systèmes d'information : de l'intérêt de combiner étude de cas et simulation multi-agents". Systèmes d'Information et Management, 23(3), 127-153.

Merminod, V., Rowe, F. (2018). «Identification de configurations par la méthode Fuzzy set Qualitative Comparative Analysis : Illustration par la contribution de la technologie PLM au respect du temps de développement». Systèmes d'Information et Management, 23(3), 71-97.

Pallud, J., Godé, C., De Corbière, F. (2021). "L'importance de la contribution dans la revue SIM ». Systèmes d'Information et Management, 26(1), 3-7.

Pascal, A., Aldebert, B., Rouziès, A. (2018). «Les méthodes mixtes en systèmes d'information : enjeux épistémologiques et méthodologiques ». Systèmes d'Information et Management, 23(3), 99-126.

Pigneur, Y. (2012). «Lancement d'une nouvelle rubrique 'opinions' ». Systèmes d'Information et Management, 17(4), 3-7 
Rivard, S. (2020). "Theory building is neither an art nor a science. It is a craft." Journal of Information Technology, May 2020.

Rowe, F. (2014). "What literature review is not: diversity, boundaries and recommendations." European Journal of Information Systems, 23(3), 241-255.
Templier, M., Paré, G. (2015). "A framework for guiding and evaluating literature reviews." Communications of the Association for Information Systems, 37(6), 112-137

Walsh, I., Renaud, A. (2017). "Reviewing the literature in the IS field: Two bibliometric techniques to guide readings and help the interpretation of the literature." Systèmes d'Information et Management, 22(3), 75-115. 